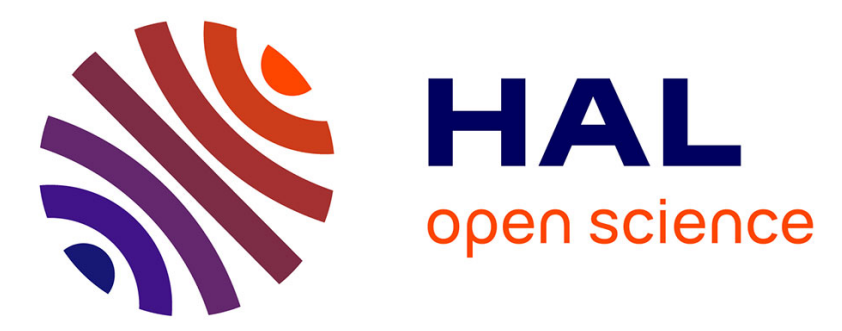

\title{
Generic 4D flight guidance based on differential flatness
}

Wen Chi Lu, Lili Duan, Thierry Miquel, Felix Mora-Camino

\section{To cite this version:}

Wen Chi Lu, Lili Duan, Thierry Miquel, Felix Mora-Camino. Generic 4D flight guidance based on differential flatness. ACA 2007, 17th IFAC Symposium on Automatic Control in Aerospace, Jun 2007, Toulouse, France. pp 353-358, 10.3182/20070625-5-FR-2916.00061 . hal-00938155

\section{HAL Id: hal-00938155 \\ https://hal-enac.archives-ouvertes.fr/hal-00938155}

Submitted on 27 May 2014

HAL is a multi-disciplinary open access archive for the deposit and dissemination of scientific research documents, whether they are published or not. The documents may come from teaching and research institutions in France or abroad, or from public or private research centers.
L'archive ouverte pluridisciplinaire HAL, est destinée au dépôt et à la diffusion de documents scientifiques de niveau recherche, publiés ou non, émanant des établissements d'enseignement et de recherche français ou étrangers, des laboratoires publics ou privés. 


\title{
GENERIC 4D FLIGHT GUIDANCE BASED ON DIFFERENTIAL FLATNESS
}

\author{
Wen Chi Lu ${ }^{1}$, Lili Duan ${ }^{1}$, Thierry Miquel ${ }^{1,2}$ and Felix Mora-Camino ${ }^{1,3}$ \\ ${ }^{1}$ LARA, ENAC, 7 avenue Edouard Belin, 31055 Toulouse, France \\ 2 DSNA/DTI/R\&D, 7 avenue Edouard Belin, 31055 Toulouse, France \\ ${ }^{3}$ LAAS du CNRS, 7 avenue du Colonel Roche, 31077, Toulouse, France
}

\begin{abstract}
With the increase of traffic density, it appears of interest to design more versatile guidance systems for civil transportation aircraft. In this paper, a new approach is proposed to design a guidance system able to achieve $4 \mathrm{D}$ trajectory tracking. Flight guidance dynamics are shown to be implicit differentially flat with respect to the inertial position of an aircraft. To overcome the numerical difficulty implied by this implicit property, artificial neural networks are introduced to capture the differential relationship between guidance parameters and attitude control inputs. A corrective term is also introduced to take into account the effects of model approximation, neural network errors and disturbances.
\end{abstract}

Keywords: flight control, tracking, nonlinear control, neural networks, corrective feedback.

\section{INTRODUCTION}

The sustained increase of air transportation during the last decades has led to severe air traffic saturation and environmental problems and new needs for aircraft guidance applications have appeared: traffic avoidance and relative guidance manoeuvres, formation flight, continuous descent approaches, etc. It has become more and more difficult to integrate these new complex functions within the classical mode-based approach of guidance manoeuvres where complex manoeuvres are performed through the scheduling of elementary guidance modes. Until recently few studies dealing with what seems more profitable and safe in that case: a full trajectory-based approach of flight guidance, have been published. In this communication, a contribution to this question, based on differential flatness (Fliess, et a.l, 1995), is proposed.

Some authors have investigated the differential flatness of conventional aircraft dynamics (Martin, 1992; Lavigne, et al., 2003), although none of them has considered separately the flatness property of the flight guidance and the attitude dynamics of a rigid aircraft.

A neural guidance control structure is proposed to perform trajectory-tracking manoeuvres. The inertial position of an aircraft is taken as a differential flat output for its flight guidance dynamics (FGD), giving way to their static inversion. Since this differential flatness property is implicit, a neural network is introduced, as a numerical device, to deal with the inversion of the guidance dynamics. Once conveniently structured and trained, the neural network is able to generate in real time directives to conventional autopilot systems concerned with attitude and engine regime control so that the reference trajectory can be tracked. In order to compensate modelling errors and external disturbances, an additional feedback loop must be introduced. All these issues, as well as the robustness question with respect to modelling errors and external perturbations, are discussed in this paper.

\section{DIFFERENTIAL FLATNESS}

Here two definitions of flatness are considered: One relative to systems for which causal relationships are displayed analytically, it is called explicit flatness and another one, called implicit flatness, where the causal relationships are introduced through implicit functions. The term differential is dropped in the remaining of this paper to avoid unnecessary cumbersome denominations.

Definition 1: A general nonlinear system given by:

$$
\underline{\dot{X}}=F(\underline{X}, \underline{U}), \underline{X} \in \mathbf{R}^{n}, \underline{U} \in \mathbf{R}^{m}
$$

where $F$ is a smooth mapping, is said explicitly flat with respect to the output vector $\underline{Z}$, if $\underline{Z}$ is an 
$m^{\text {th }}$ order vector which can be expressed analytically as a function of the current state, the current input and its derivatives, while the state and the input vectors can be expressed analytically as a function of $\underline{Z}$ and a finite number of its derivatives. Then there exists smooth mappings $G_{X}, G_{U}$, and $G_{Z}$ such as:

$$
\begin{aligned}
& \underline{Z}=G_{Z}\left(\underline{X}, \underline{U}, \ldots, \underline{U}^{(p)}\right) \\
& \underline{X}=G_{X}\left(\underline{Z}, \underline{\dot{Z}}, \ldots, \underline{Z}^{(q)}\right) \\
& \underline{U}=G_{U}\left(\underline{Z}, \underline{Z}, \ldots, \underline{Z}^{(q+1)}\right)
\end{aligned}
$$

where $p$ and $q$ are integer numbers. Vector $Z$ is called a flat output for the nonlinear system given by equation (1). Although today there is no systematical way to determine flat outputs and eventually to prove its uniqueness, the flat outputs usually possess some physical meaning.

The explicit flatness property is of particular interest for the solution of control problems when physically meaningful flat outputs can be related with their objectives. In many situations, the control problem can be formulated as a flat output trajectory following problem. In general, for these cases, the flat output of equation (2a) can be reduced, through state transformation, to a function of a single argument, the new system state itself:

$$
\underline{Z}=G_{Z}(\underline{X})
$$

However, for many systems, no complete analytical models are available to describe their full dynamics. Some of their components make use of input-output numerical devices derived both from theory and from experimental data. In these cases, available theory provides in general the main mathematical properties of these implicit functions while experimental data is used to build accurate input-output numerical devices. This happens for instance when flight dynamics modeling is considered either for control or simulation purposes, since in practice the aerodynamic coefficients are obtained through interpolation across a complex set of look-up tables.

Definition 2: A nonlinear system given by a general implicit $\mathrm{n}^{\text {th }}$ order state representation:

$$
F(\underline{X}, \underline{\dot{X}}, \underline{U})=0, \underline{X} \in R^{n}, \underline{U} \in R^{m}
$$

where $F$ is a regular implicit mapping with respect to $\underline{\dot{X}}$, is said implicitly flat over an interior nonempty domain $\Delta \subseteq R^{n+m}$ if it is possible to find an $m^{t h}$ order vector $\underline{Z}$ which meets conditions of equations (2) and condition:

$$
G\left(\underline{X}, \underline{U}, \underline{Z}, \underline{\dot{Z}}, \ldots, \underline{Z}^{(r)}\right)=\underline{0}
$$

where $G$ is locally invertible over $\Delta$ with respect to $\underline{X}$ and $\underline{U}$. Here $r$ is an integer.
Again, vector $\underline{Z}$ is said to be a flat output. The invertibility of $G$ is guaranteed if the determinant of the Jacobian of $G$ is not zero according to the theorem of implicit functions. Equations (4) and (5) imply that given a trajectory of the flat output $\underline{Z}$, it is possible to map it numerically into the input space to derive corresponding control signals so that one of the more helpful properties of explicitly flat systems is still maintained.

\section{ANALYSIS OF FLIGHT GUIDANCE DYNAMICS}

\subsection{A model of FGD}

In this study, only the guidance dynamics of transportation aircraft are of concern since it is assumed that the considered aircraft are equipped with basic autopilots, which deal efficiently with their fast dynamics and control their attitude $(\theta, \phi, \beta)$ as well as their thrust regimes $\left(N_{1}\right)$.

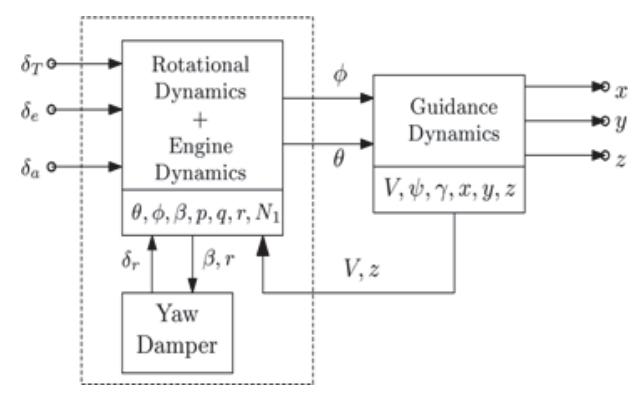

Fig.1. Aircraft Flight Dynamics Structure

Here: $V_{a}$ is the airspeed; $V$ is the inertial speed; $x, y$ and $z$ are the aircraft coordinates in the inertial frame; $\beta$ is the sideslip angle; $p, q$ and $r$ are the angular rates in the body-axis frame; $\phi, \theta$ and $\psi$ are the Euler angles (roll, pitch, and yaw); $\gamma$ is the flight-path angle; $\delta_{a}$ and $\delta_{e}$ are the aileron and elevator settings; $\delta_{T}$ is the throttle setting.

It is also supposed that their lateral fast control loop can achieve perfect coordinated-turns, so that $\beta$ is supposed to remain very small. Flight variables $\theta$, $\phi$ and $N_{1}$, which are the outputs for the fast dynamics around the aircraft body frame when controlled by a basic autopilot, are taken here as the inputs for the guidance dynamics. Figure 1 displays the resulting view for the whole flight dynamics. Then, in steady wind conditions (Etkin and Reid, 1996), the flight guidance dynamics can be expressed in the aerodynamics reference frame as:

$$
\left.\begin{array}{l}
\dot{x}=V_{a} \cos \psi \cos \gamma+w_{x} \\
\dot{y}=V_{a} \sin \psi \cos \gamma+w_{y} \\
\dot{z}=-V_{a} \sin \gamma+w_{z}
\end{array}\right\}
$$

with

$$
V_{a}=\sqrt{\left(\dot{x}-w_{x}\right)^{2}+\left(\dot{y}-w_{y}\right)^{2}+\left(\dot{z}-w_{z}\right)^{2}}
$$


and

$$
\begin{gathered}
\dot{V}_{a}=\frac{-D+T \cos \alpha-m g(-\cos \alpha \sin \theta+\sin \alpha \cos \phi \cos \theta)}{m} \\
\dot{\gamma}=\frac{L \cos \phi+T \sin \alpha-m g(\sin \alpha \sin \theta+\cos \alpha \cos \phi \cos \theta)}{m V_{a}}
\end{gathered}
$$

Considering coordinated-turn motions, the heading rate can be related to the bank angle $\phi$ through the following relation:

$$
\dot{\psi}=\left(\frac{g}{V}\right) \tan \phi
$$

The drag $D$ and lift $L$ are respectively taken as functions of altitude $z$, airspeed $V_{a}$ and angle-ofattack, while the thrust $T$ is taken as a function of altitude $z$, airspeed $V_{a}$ and engine regime $N_{1}$ for a jet:

$$
D=D\left(z, V_{a}, \alpha\right), L=L\left(z, V_{a}, \alpha\right), T=T\left(z, V_{a}, N_{1}\right)
$$

For local guidance purposes, the flight-path angle $\gamma$ is usually taken as the control parameter. Meanwhile, through the coordinate transformation, the angle-ofattack $\alpha$ can be expressed as a complex function of $\theta, \phi$, and $\gamma$. Once the bank angle is considered to be small enough, this relation can be reduced to:

$$
\alpha=\theta-\gamma
$$

\subsection{FGD flatness property}

The state variables $V_{a}, \gamma$ and $\psi$, can be expressed as functions of the inertial position of the aircraft and its derivatives. We have (6-b) and:

$$
\gamma=-\sin ^{-1}(\dot{z} / V), \quad \psi=\tan ^{-1}(\dot{y} / \dot{x})
$$

The control variables satisfy the following relations:

$$
\begin{gathered}
\dot{V}_{a}-\frac{-D+T \cos \alpha-m g(-\cos \alpha \sin \theta+\sin \alpha \cos \phi \cos \theta)}{m}=0 \\
\dot{\gamma}-\frac{L \cos \phi+T \sin \alpha-m g(\sin \alpha \sin \theta+\cos \alpha \cos \phi \cos \theta)}{m V_{a}}=0 \\
\text { and } \quad \dot{\psi}-\left(\frac{g}{V}\right) \tan \phi=0
\end{gathered}
$$

where $\alpha$ can be replaced by a function of $\phi, \theta$, and $\gamma$ using equation (9).

The following notations are adopted for the position of the center of gravity of the aircraft and for the guidance inputs:

$$
\underline{Z}=(x, y, z)^{T} \text { and } \underline{U}=\left(\theta, \phi, N_{1}\right)
$$

Once the variables in equations (11) are replaced by their expressions in $\underline{Z}$ and its first and second derivatives, these equations can be rewritten as:

$$
G_{N_{1}}(\underline{Z}, \underline{\dot{Z}}, \underline{\ddot{Z}}, \underline{U})=0, G_{\theta}(\underline{Z}, \underline{\dot{Z}}, \underline{\ddot{Z}}, \underline{U})=0, G_{\phi}(\underline{Z}, \underline{\dot{Z}}, \underline{\ddot{Z}}, \underline{U})=0
$$

We get three implicit functions which are locally invertible with respect to the input vector since for normal flight conditions the determinant of their Jacobian is not zero (Lu, 2005). Then, $\underline{Z}=(x, y, z)^{T}$ is a flat output vector for the considered flight guidance dynamics. The time evolution of these flat outputs represents the trajectory followed by the center of gravity of the aircraft. Then according to the above theory, from the knowledge of this $4 \mathrm{D}$ trajectory, it should be possible to find the corresponding input history .

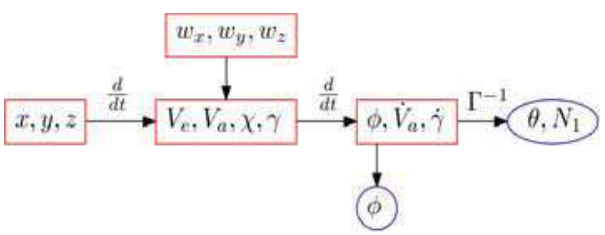

Fig.2. Flatness propagation through guidance dynamics

\section{THE PROPOSED CONTROL STRUCTURE}

\subsection{Neural Network Open Loop Control}

As a consequence of the above flatness property, given a smooth reference trajectory for the flat outputs :

$$
\underline{Z}_{c}(\tau)=\left(x_{c}(\tau), y_{c}(\tau), z_{c}(\tau)\right)^{T}, \tau \in\left[t_{0}, t\right]
$$

The corresponding reference input values at the instant $t, U_{c}(t)=\left(\phi_{c}(t), \theta_{c}(t), N_{1 c}(t)\right)^{T}$, are solutions of the set of equations:

$$
\left.\begin{array}{l}
G_{N_{1}}\left(\underline{Z}_{c}(t), \underline{\underline{Z}}_{c}(t), \underline{\underline{Z}}_{c}(t), \underline{U}_{c}(t)\right)=0, \\
G_{\theta}\left(\underline{Z}_{c}(t), \underline{\underline{Z}}_{c}(t), \underline{\underline{Z}}_{c}(t), \underline{U}_{c}(t)\right)=0 \\
G_{\phi}\left(\underline{Z}_{c}(t), \underline{\dot{Z}}_{c}(t), \underline{\underline{Z}}_{c}(t), \underline{U}_{c}(t)\right)=0
\end{array}\right\}
$$

where $\underline{Z}_{c}(t), \underline{\dot{Z}}_{c}(t)$ and $\underline{\ddot{Z}}_{c}(t)$ are the current reference parameters. Since it seems quite difficult to get an on-line numerical solution to this set of implicit equations, it appears of interest to build a neural network with this objective. Once correctly trained, this neural network should be an efficient computing device where the inputs are provided by the tracked trajectory, while the outputs of the neural network are the corresponding flight control parameters. Multilayer neural networks have been shown to be able to perform function approximation with the adequate selection of a neural network structure associated to a learning process (Haykin, 1994).

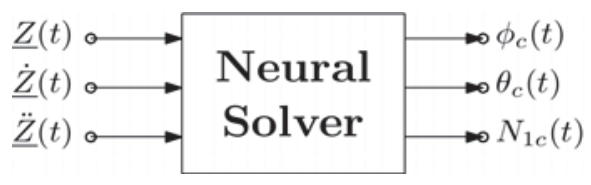

Fig. 3. neural reference input generator for autopilot

In order to achieve an acceptable accuracy and a sufficient generalization capability, a large amount of 
training data is necessary. Here the training data is composed of a set of trajectories for $\underline{Z}$ and $\underline{U}$ which can be provided from either flight test data or even from commercial flight data in which maneuvers are either performed manually or with an autopilot engaged in basic attitude-holding modes (no guidance loop must be active at that time).

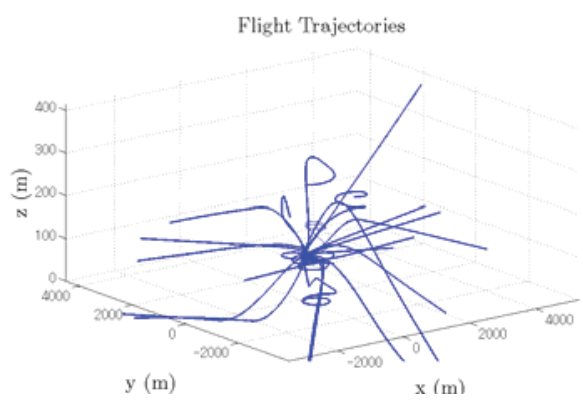

Fig.4. A set of training trajectories

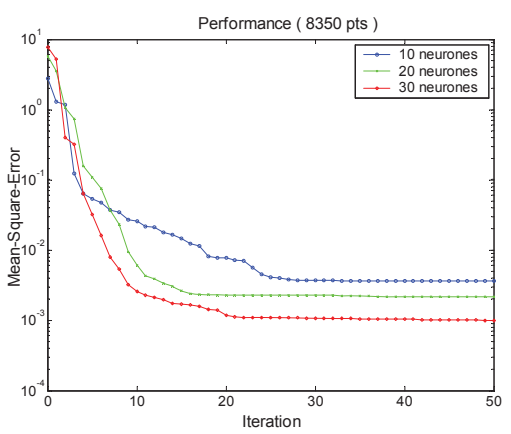

Fig.5. Training performance with different number of neurons in the hidden layer

Since for modern aircraft onboard navigation systems are able to estimate with good accuracy the current aircraft position, inertial speed and wind speed, their records can be used as a basis for the training of the neural network.

\subsection{Closed Loop Tracking}

The direct application of the above approach should bring a guidance drift, which will grow uncontrolled with time (see figs.6-a and 6-b) since the resulting control policy is basically of the open loop class. Modelling errors (mainly with respect to the aerodynamic and thrust effects), neural network generalization errors and external perturbations (winds and non standard atmosphere) will be the main causes of this drift.
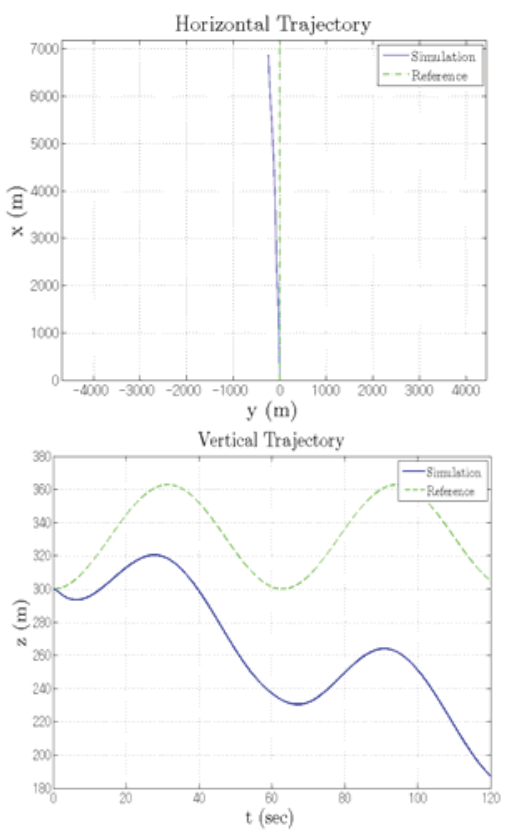

Fig.6. Example of open loop performances

Then it appears that a closed loop must be added to this structure to guarantee guidance accuracy. Figure 6 displays a possible flight guidance control structure, which integrates a neural network solver and a corrective loop. To design this corrective loop a model of the dynamics of the drift is adopted. The guidance dynamics equations can be written as:

$$
\underline{\dot{X}}=f(\underline{X}, \underline{Z}, \underline{U})
$$

with

$$
\begin{aligned}
& \underline{X}=\left(V_{a}, \gamma, \psi\right)^{\prime}, \quad \underline{Z}=(x, y, z)^{\prime}, \underline{U}=\left(\theta, \phi, N_{1}\right) \\
& \text { and } \quad \underline{\dot{Z}}=g(\underline{X})+\underline{w} \\
& \text { with } \quad \underline{w}=\left(w_{x}, w_{y}, w_{z}\right)^{\prime}
\end{aligned}
$$

where $\underline{w}$ is the wind vector expressed in the local Earth frame. Then, starting from nominal values $V_{a}(0), \gamma(0)$ and $\psi(0)$, a first order approximation of the drift dynamics is given by :

$$
\begin{aligned}
\underline{\delta \dot{X}}=\frac{\partial f}{\partial \underline{X}} \frac{\delta X}{\delta}+\frac{\partial f}{\partial \underline{U}}(\underline{X}, \underline{U}) \underline{\delta U} \\
\text { and } \quad \underline{\delta \dot{Z}}=\frac{\partial g}{\partial \underline{X}}(\underline{X}) \underline{\delta X}+\underline{w}
\end{aligned}
$$

Assume that around the reference conditions, the output error of the neural network is such as:

$$
R N(\underline{e}+\underline{\delta e})=R N^{*}(\underline{e})+G(\underline{Z}(0), \underline{X}(0)) \underline{\delta e}+\underline{\sigma}
$$

where $R N^{*}(e)$ is the unknown exact output for input $e, \delta e$ is the input deviation from reference and $\sigma$ is a bias. Then the flight control input error is such as :

$$
\underline{\delta U}=K(\underline{Z}(0), \underline{X}(0)) \underline{\delta e}+\underline{\sigma}
$$

where in general the gain matrix $K$ is such as : 
$S(K)=\left[\begin{array}{ccccccc}0 & 0 & 0 & 0 & * & * & * \\ * & * & * & * & 0 & 0 & 0 \\ * & * & * & * & 0 & 0 & 0\end{array}\right]$ with $\operatorname{rank}(K)=3$

Since $\frac{\partial g}{\partial \underline{X}}$ is non singular $\left(\left|\frac{\partial g}{\partial \underline{X}}\right|=V_{a}^{2} \cos ^{3} \gamma\right)$, eliminating $\underline{\delta X}$, we get the following fully controllable output dynamics:

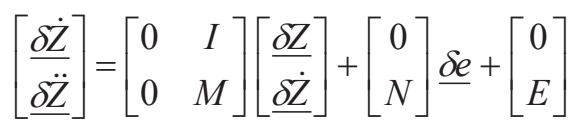

with:

$$
\begin{gathered}
M=\frac{\partial g}{\partial \underline{X}} \frac{\partial f}{\partial \underline{X}}\left(\frac{\partial g}{\partial \underline{X}}\right)^{-1} \quad N=\frac{\partial g}{\partial \underline{X}} \frac{\partial f}{\partial \underline{U}} G \\
\underline{E}=\frac{\partial g}{\partial \underline{X}}\left(-\frac{\partial f}{\partial \underline{X}}\left(\frac{\partial g}{\partial \underline{X}}\right)^{-1} \underline{w}+\frac{\partial f}{\partial \underline{U}} \underline{\sigma}\right)
\end{gathered}
$$

Now, assuming that $w$ and $\sigma$ are slow varying parameters, we get the fully observable dynamics from $\delta Z$ :

$$
\left[\begin{array}{l}
\frac{\delta \dot{Z}}{\dot{\ddot{Z}}} \\
\dot{\dot{E}}
\end{array}\right]=\left[\begin{array}{ccc}
0 & I & 0 \\
0 & M & I \\
0 & 0 & 0
\end{array}\right]\left[\begin{array}{l}
\frac{\delta Z}{\underline{\delta}} \\
\underline{E}
\end{array}\right]+\left[\begin{array}{c}
0 \\
N \\
0
\end{array}\right] \underline{\delta e}
$$

and it is possible to get an on line estimation of $E(t)$, written $\hat{E}(t)$ and given by :

$$
\begin{gathered}
\underline{\hat{E}}=\int_{0}^{t} L_{3}(\underline{\delta Z}(\tau)-\delta \hat{Z}(\tau)) d \tau \\
\text { with }\left[\begin{array}{c}
\frac{\delta \dot{\hat{Z}}}{\dot{\hat{\dot{Z}}}} \\
\frac{\delta}{\hat{\dot{E}}}
\end{array}\right]=\left[\begin{array}{ccc}
0 & I & 0 \\
0 & M & I \\
0 & 0 & 0
\end{array}\right]\left[\begin{array}{l}
\frac{\delta \hat{Z}}{\delta \hat{\dot{Z}}} \\
\hat{\hat{E}}
\end{array}\right]+\left[\begin{array}{c}
0 \\
N \\
0
\end{array}\right] \underline{\delta e}+\left[\begin{array}{l}
L_{1} \\
L_{2} \\
L_{3}
\end{array}\right](\underline{\delta Z}-\underline{\delta \hat{Z}})
\end{gathered}
$$

Then a closed loop control law such as :

$$
\underline{\delta e}(t)=-F_{\delta Z} \underline{\delta Z}(t)-F_{\delta \dot{Z}} \underline{\delta \dot{Z}}(t)-F_{E} \int_{0}^{t}(\underline{\delta Z}(\tau)-\underline{\delta \hat{Z}}(\tau)) d \tau
$$

where matrix $\left[\begin{array}{cc}0 & I \\ -N F_{\delta Z} & M-N F_{\delta \dot{Z}}\end{array}\right]$ is stable and:

$$
N F_{E}=I
$$

so that the condition $\lim _{t \rightarrow+\infty} \quad \underline{\delta Z}(t)=\underline{0} \quad$ is achieved.

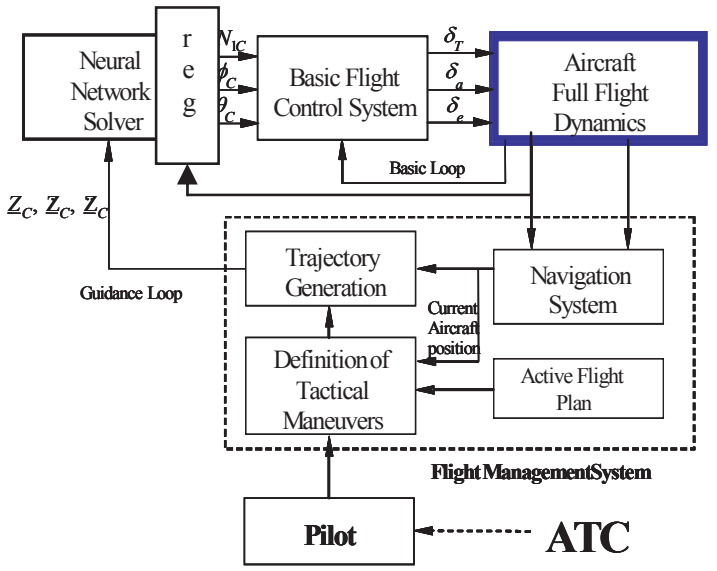

Fig. 7. A possible flight guidance control structure

\section{SIMULATION RESULTS}

In this study, the conventional Error-BackPropagation neural network with only one hidden layer has been selected to perform the inversion of flight guidance dynamics, although other neural network structures have been investigated. The structure of the retained neural network comprises five inputs nodes, empirically determined number of thirty neurons with a hyperbolic tangent activation function in the hidden layer, and three output nodes with linear transfer functions. The seven inputs are altitude, three components of inertial velocity and three components of inertial acceleration. The three outputs are pitch command $\theta$, roll command and the engine command $\Omega$.

A simulation model of a light aircraft (AeroSim, 2003) with a piston-propeller engine and a simple PID controller for attitude holding is used for the generation of training data and is also used for validation purpose.

The Guidance closed loop gains have been chosen so that they satisfy relations (28). However, for $F_{\delta Z}$ and $F_{\delta \dot{Z}}$ a large choice of values remains available, so that it is quite easy to deal with additional requirements, such as robustness, at this level.

The following figures represent first tracking maneuvers in the horizontal plane (figures 8), then in the vertical plane (figures 9) and finally in the three dimensional space (figures 10).

The different results obtained show the performances in terms of accuracy and robustness of the proposed control solution.
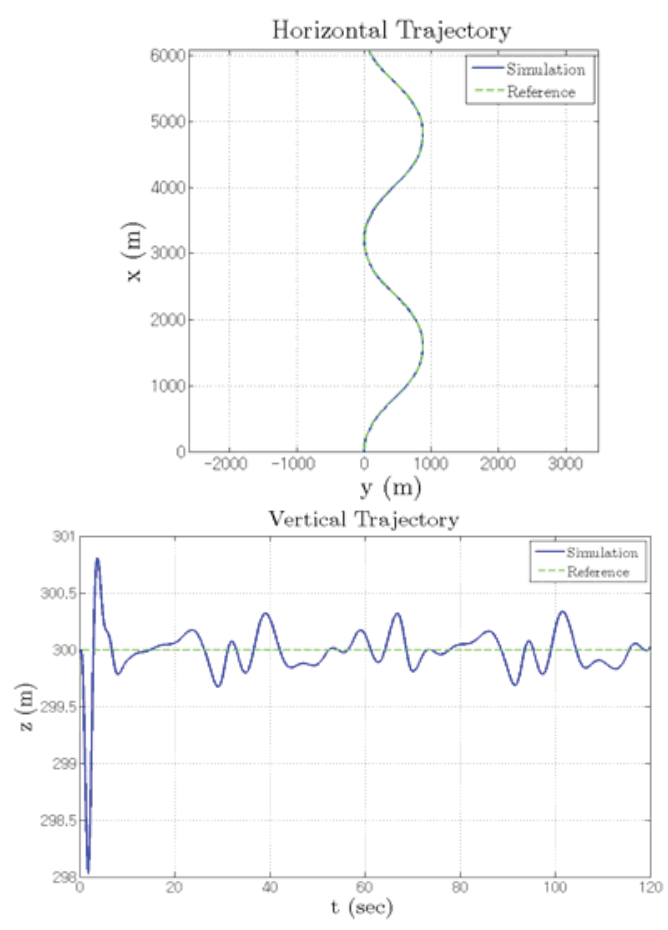

Fig. 8. Trajectories and drift for $V=60 \mathrm{~m} / \mathrm{s}, \gamma=0^{\circ}$ 

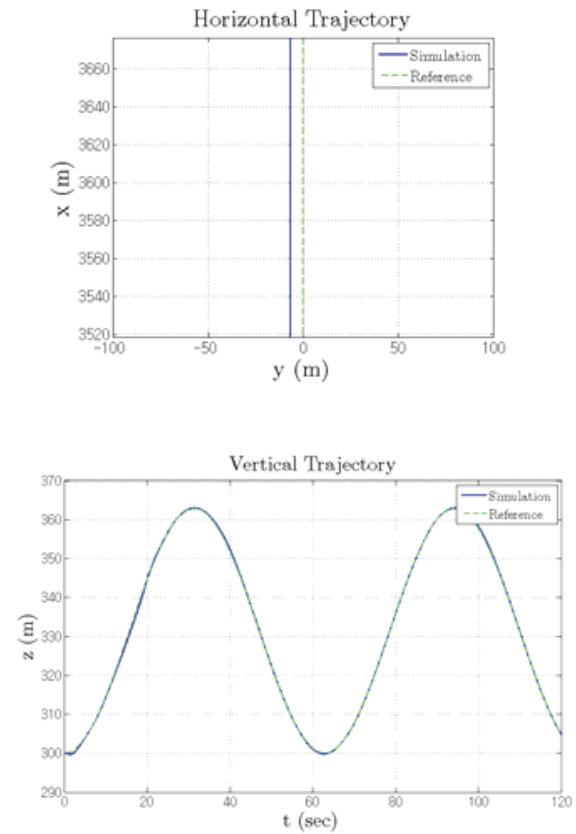

Fig.9. Trajectories and drift for $V=60 \mathrm{~m} / \mathrm{s}, \gamma=$ $3 * \sin (0.1 \mathrm{t})$ and $\psi=0$
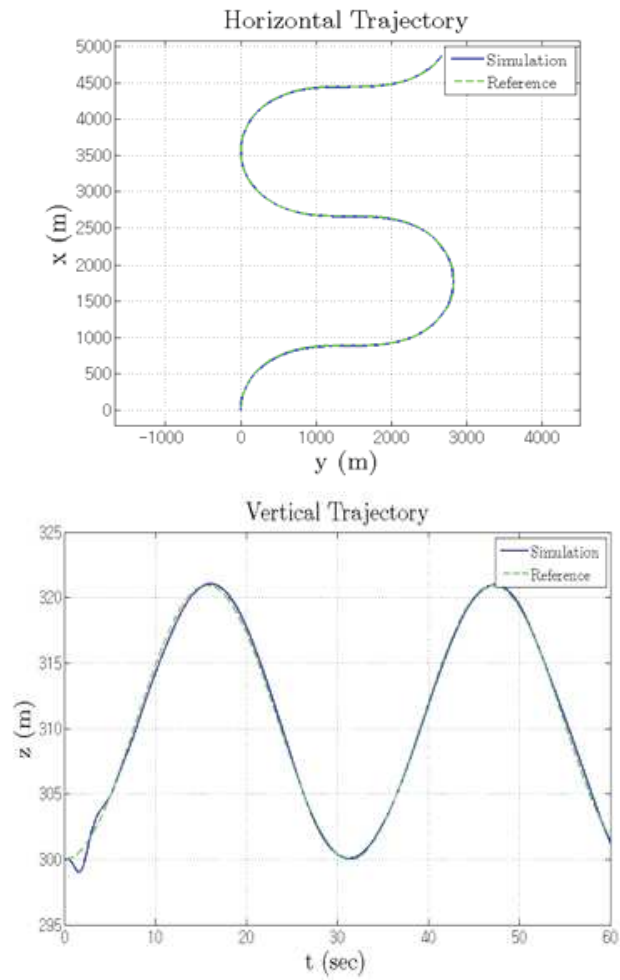

Fig.10. Trajectories and drift for $V=60 \mathrm{~m} / \mathrm{s}, \gamma=$ $2 * \sin (0.1 \mathrm{t})$ and $\psi=90 * \sin (0.05 \mathrm{t})$

\section{CONCLUSIONS}

This paper opens the way to new developments in the fields of flight guidance systems. Here, differential flatness theory has provided a sound basis for the introduction of neural networks technology within the architecture of flight guidance systems. The resulting approach appears to provide a possible basis for the development of a generic guidance systems which should allow accurate trajectory tracking maneuvers, either classical or not. The training of the neural network generator for a given aircraft should insure that the generic guidance system is compatible with its basic autopilot system. Many issues related with the training process of the neural network devoted to guidance and with the robustness of the corrective term with respect to disturbances and modeling errors, remain open.

\section{REFERENCES}

Etkin, B. and L.R. Reid (1996). Dynamics of FlightStability and Control. John Wiley \& Sons. New York, NY.

Fliess,M., J. Lévine, P. Martin, and P. Rouchon 1995). Flatness and defect of non-linear systems: theory and examples. International Journal of Control, Vol. 61, No. 6, 1995, pp. 1327-1361.

Haykin, S. (1994). Neural Networks, a Comprehensive Foundation. Macmillan Publishing Company, New Jersey.

Lavigne, L., F. Cazaurang and B. Bergeon (2003). Modelling of Longitudinal Disturbed Aircraft Model by Flatness Approach. AIAA Guidance, Navigation, and Control Conference. Austin, Texas.

Lu, W.C., F. Mora-Camino, M. de Coligny and K. Achaibou (2004). Flight Mechanics and Differential Flatness. Dynamics and Control Conference. Ilha Solteira, Brasil, pp. 830-839, 2004.

Lu, W.C. (2005). Contribution au Suivi Automatique de Trajectoires par un Avion: Commande Plate et Réseaux de Neurones. Université Toulouse 2. $\mathrm{PhD}$ Thesis, Toulouse.

Martin, P. (1992). Contribution à l'étude des systèmes differentiellement plats. Ecole des Mines de Paris. Ph.D. Dissertation, Paris.

Unmanned Dynamics, LLC,(2003).Aerosym Blockset User's Guide, (2003). Version 1.1.http://www.udynamics.com. 